\title{
VEDAG - historischer Dachverband mit gesundheitspolitischem Weitblick
}

Christoph Ramstein,

Co-Präsident VEDAG

1 Brunner HH. 100 Jahre FMH. Schweiz Ärztezeitung. 2001:82 (51-52):2659-65.

2 Peltenburg M, Kernen $\mathrm{H}$, Schneider P, von Below GC, Waldis G, Vogel HA, et al. Qualität ein Zusammenspiel aller Kräfte im ärztlichen Umfeld. Schweiz Ärztezeitung. 2005;86(18):1055-9.

3 VEDAG. Gesundheitspolitik aus der Optik der Schweizer Ärztinnen und Ärzte. Schweiz Ärztezeitung. 2004;85(30):1531-4.

Korrespondenz:

Dr. med. Christoph Ramstein

Marktgasse 39

CH-4600 Olten

christoph.ramstein@hin.ch

\begin{abstract}
Historisches
Die Wurzeln des VEDAG gehen auf den 1870 gegründeten Ärztlichen Centralverein zurück, der nebst den 17 kantonalen Ärztegesellschaften der Deutschschweiz auch den Kanton Neuenburg umfasste.

Unter der Ägide des Ärztlichen Centralvereins (ab 1931 VEDAG genannt) begann ab 1874 ein langwieriger Prozess, der unter Zusammenschluss mit dem SMSR (Société Médicale de la Suisse Romande) am 15. Dezember 1901 zur Gründung der heutigen FMH geführt hat. Das historisch und kulturell bedingte Spannungsfeld RomandieDeutschschweiz hat die Geschichte der Ärzteschaft bis auf den heutigen Tag geprägt [1].
\end{abstract}

\section{Aktivitäten und Anliegen des VEDAG im 20. Jahrhundert ...}

In den letzten 20 Jahren befasste sich der VEDAG mit folgenden Schwerpunktthemen:

\section{WZW-Verfahren}

Die WZW-Verfahren wurden vom damaligen KSK (Konkordat Schweizerischer Krankenversicherer) in den einzelnen Kantonen sehr unterschiedlich gehandhabt. Die Tragweite dieser WZW-Verfahren für die betroffenen Kolleginnen und Kollegen wurde weder von der Ärztekammer noch vom ZV erkannt. Der VEDAG unterstützte daher vermehrt private Organisationen wie die Consano (www.consano.ch), die sich vertieft mit diesen Problemen befassen.

\section{Ärztliche Medikamentenabgabe}

Der VEDAG setzt sich im Interesse der Patienten für den Erhalt der Wahlfreiheit der bestehenden Abgabekanäle ein.

\section{Koordination der kantonalen Tarifverhandlungen}

Im Hinblick auf die Einführung von TARMED wurde auf Initiative des VEDAG die G7 (später KKA) gegründet. Sie koordiniert und unterstützt alle Kantone in den Tarifverhandlungen. In Zusammenarbeit mit der NewIndex AG entstanden zudem die TrustCenters zwecks Sicherung der Datenparität.

\section{... und im 21. Jahrhundert}

Als Dachverband ist der VEDAG bestrebt, auch weiterhin die standespolitischen Grundanliegen seiner Basis sowohl in der Ärztekammer wie auch in der neu gegründeten Delegiertenversammlung der FMH einzubringen. Häufig nimmt er auch die Rolle eines «advocatus diaboli» wahr und gerät dadurch in Opposition zu anderen Gruppierungen, die zu stark der Besitzstandwahrung verhaftet sind. Die Kantonalpräsidenten sind direkt mit den praktischen Problemen der Gesundheitspolitik konfrontiert, die im VEDAG lösungsorientiert diskutiert werden. Eine der Hauptanliegen des VEDAG ist die ärztliche Qualitätspolitik. An der Ärztekammer 2005 wurde das an der Klausurtagung 2003 bearbeitete Projekt mitsamt der Arbeitsgruppe AGQ I des VEDAG in die DDQ der FMH übergeführt [2]. Im Herbst 2007 entstand das Arbeitspapier «Qualität in der ärztlichen Praxis: Staats- versus Wettbewerbsmodell», das nun in der Arbeitsgruppe AGQ II weiterverfolgt wird.

In früheren Klausuren entstanden weitere Positionspapiere [3].

\section{Zeitschrift «Sprechstunde» und Magazin «Politik + Patient»}

Seit 1971 erscheint unter dem Patronat des VEDAG die «Sprechstunde», das offizielle PatientInnenmagazin der Ärztinnen und Ärzte (www. sprechzimmer.ch) mit Informationen zu aktuellen medizinischen und gesundheitspolitischen Themen.

Seit 2006 gibt der VEDAG zudem das Politmagazin «Politik + Patient» heraus, das sich vertieft mit den gesundheitspolitischen Anliegen der Ärzteschaft befasst und sich einerseits an unsere Patienten sowie andererseits an unsere kantonalen und eidgenössischen Parlamentarier richtet. 